\title{
Postoperative translation of the upper instrumented vertebra in thoracic adolescent idiopathic scoliosis
}

\author{
Katsuhisa Yamada, MD, PhD, ${ }^{1}$ Hideki Sudo, MD, PhD, ${ }^{1}$ Kiyoshi Kaneda, MD, PhD, ${ }^{2}$ \\ Yasuhiro Shono, MD, PhD, ${ }^{3}$ Yuichiro Abe, MD, PhD, ${ }^{4}$ and Norimasa Iwasaki, MD, PhD ${ }^{1}$
}

${ }^{1}$ Department of Orthopaedic Surgery, Hokkaido University Hospital; ${ }^{2}$ Ogasawara Clinic Sapporo Hospital; ${ }^{3}$ Department of Orthopaedic Surgery, Japan Community Health Care Organization Hokkaido Hospital, Sapporo; and ${ }^{4}$ Department of Orthopaedic Surgery, Eniwa Hospital, Eniwa, Hokkaido, Japan

\begin{abstract}
OBJECTIVE The aim of this retrospective study was to analyze the influence of upper instrumented vertebra (UIV) translation from the $\mathrm{C} 7$ plumb line (C7PL) on the long-term postoperative results of patients with main thoracic (MT) adolescent idiopathic scoliosis (AIS).

METHODS Twenty-five patients had been treated surgically for AIS with a Lenke type 1 curve and had been followed up for a mean period of 18.2 years. Radiographic parameters, pulmonary function measurements, and clinical outcomes were compared between the patients $(n=15)$ with UIV translation $<20 \mathrm{~mm}$ and those $(n=10)$ with UIV translation $\geq 20$ $\mathrm{mm}$ at the final follow-up. Correlations between UIV translation and radiographic or pulmonary function parameters were analyzed.
\end{abstract}

RESULTS Patients with $\geq 20 \mathrm{~mm}$ UIV translation at the final follow-up had a significantly larger preoperative UIV translation than that in the patients with $<20 \mathrm{~mm}$ UIV translation at follow-up. The former group also had a significantly lower correction rate of the MT curve, higher chest cage ratio, and lower radiographic shoulder height $(p=0.01,0.005$, and 0.025 , respectively) at the final follow-up. The Scoliosis Research Society (SRS)-30 Questionnaire scores were equivalent between the two groups. Correlation analysis showed that the following parameters were significantly associated with UIV translation: MT curve correction rate $(r=-0.481, p=0.015)$, chest cage ratio $(r=0.673, p<0.001)$, and percent-predicted forced expiratory volume in 1 second $(r=-0.455, p=0.033)$.

CONCLUSIONS The UIV translation should be considered an important factor that influences postoperative results. In MT AIS patients whose preoperative upper end vertebra (UEV) is distant from the C7PL, the UIV should be selected above the UEV to prevent large UIV translation at the postoperative follow-up.

https://thejns.org/doi/abs/10.3171/2018.6.PEDS18256

KEYWORDS adolescent idiopathic scoliosis; main thoracic curve; anterior spinal fusion; upper instrumented vertebra; UIV translation; spine

$\mathrm{T}$ HE current gold standard treatment for adolescent idiopathic scoliosis (AIS) is posterior spinal fusion (PSF) with pedicle screw instrumentation. ${ }^{6,20}$ However, three-dimensional corrections with the fusion of fewer motion segments can be established through anterior spinal fusion (ASF), ${ }^{1,4,8,16}$ which remains an attractive option for treating thoracolumbar and lumbar AIS curves.,17,21 Another potential advantage of the anterior approach to the thoracic spine is the ability to restore kyphosis within the range of the main thoracic (MT) curve., , $, 8,14,16,24$ Sudo et al. recently reported that overall radiographic findings and patient outcome measures after ASF for Lenke type 1 AIS were satisfactory at an average follow-up of 15 years, although the procedure decreased pulmonary function. ${ }^{22}$

Another potential disadvantage of ASF is the limited choice of the upper instrumented vertebra (UIV). Given the presence of the scapular arch, it is very difficult for vertebral screws to be inserted into the vertebral bodies and vertebral plates. Instrumentation levels are determined from end-to-end vertebrae on standing films in most cases because surgeons resort to a short fusion strategy in relation to the UIV with the aim of preventing additional

ABBREVIATIONS AIS = adolescent idiopathic scoliosis; $\mathrm{ASF}=$ anterior spinal fusion; $\mathrm{AVT}=$ apical vertebral translation; $\mathrm{C7PL}=\mathrm{C7}$ plumb line; CSVL = center sacral vertical line; $\mathrm{FEV}_{1}$ = forced expiratory volume in 1 second; FVC = forced vital capacity; LEV = lower end vertebra; LIV = lower instrumented vertebra; MT = main thoracic; PSF = posterior spinal fusion; PT = proximal thoracic; RSH = radiographic shoulder height; SRS = Scoliosis Research Society; UEV = upper end vertebra; UIV = upper instrumented vertebra.

SUBMITTED May 3, 2018. ACCEPTED June 6, 2018.

INCLUDE WHEN CITING Published online September 7, 2018; DOI: 10.3171/2018.6.PEDS18256. 
operation time, bleeding, muscle damage, and skin incision as well as preserving mobile spinal segments. However, no reports have evaluated the selection of the UIV in Lenke type 1 AIS curve correction using ASF. During the long-term follow-up of ASF for Lenke type 1 AIS, we have noticed that the translation of the UIV increases in some cases, which might be related to postoperative results. Hence, the purpose of this study was to assess the incidence of UIV translation after ASF in the treatment of Lenke type 1 AIS and to analyze the influence of UIV translation on radiographic parameters, pulmonary function, and clinical outcome scores. In addition, we assessed preoperative factors that might determine postoperative UIV translation. Such factors may be important for the surgical strategy in terms of fusion level determination.

\section{Methods}

This study was approved by the institutional review board of Hokkaido University Hospital. We retrospectively reviewed the data on 25 consecutive patients $(3$ males, 22 females) with Lenke type 1 MT AIS curves ${ }^{7}$ who had undergone anterior MT curve correction at our institution between 1994 and 2000. ${ }^{22}$ Study exclusion criteria consisted of the presence of syndromic, neuromuscular, and congenital scoliosis, a follow-up $<10$ years, and the presence of other thoracic scoliosis curves such as Lenke type 2 double thoracic curves. None of the patients in the study had clinical lung function issues. An independent spine surgeon collected all data. No cases were lost to follow-up. Radiographs, pulmonary function, and the Scoliosis Research Society (SRS) outcome instrument (SRS-30 Questionnaire) scores ${ }^{10,15}$ were evaluated for all patients. The SRS-30 Questionnaire scores were available only at the final follow-up. The patient records were de-identified and analyzed anonymously.

Standing long-cassette posteroanterior and lateral radiographs were evaluated for multiple parameters before and after surgery and at the final follow-up. Curve flexibility was evaluated preoperatively with supine bending radiographs. Coronal Cobb measurements of the proximal thoracic (PT) and MT curves were obtained. End vertebrae levels were determined on preoperative radiographs and were measured on subsequent radiographs to maintain consistency for statistical comparisons. ${ }^{21}$ The sagittal measurement assessed was the instrumented level. Global coronal balance was measured by the lateral displacement of the C7 plumb line (C7PL) from the center sacral vertical line (CSVL). ${ }^{3}$ For regional alignment, MT apical vertebral translation (AVT) was measured as the distance between the geometrical center of the apical vertebra and the C7PL. ${ }^{23}$ The chest cage ratio is the ratio of the thorax width (horizontal distance from the geometrical center of the apex vertebra to each outline of the left and right thoracic cage) on the left side to the thorax width on the right side. The radiographic shoulder height (RSH) is the vertical distance between the most cephalad margin of the acromion to the soft-tissue outline immediately above the acromion and a horizontal reference line, with a positive value indicating that the left side is high. ${ }^{11}$

The radiographic parameters relevant to the UIV were measured as follows: UIV translation was measured as the distance between the geometrical center of the UIV and the C7PL (Fig. 1). ${ }^{9}$ The UIV tilt was defined as the inclination of the upper endplate of the UIV from the horizontal. The coronal disc angle immediately superior to the UIV was considered the UIV disc angle. The UIV tilt and UIV disc angle were defined as negative when they opened to the preoperative convex side and as positive when they opened to the preoperative concave side, as previously reported. ${ }^{18,23}$ Sagittal balance was evaluated by measuring the absolute displacement value of the C7PL relative to the S1 posterior superior corner as the sagittal vertical axis. ${ }^{19}$ Finally, proximal junctional kyphosis was defined as the sagittal disc angle between the UIV and the proximal vertebra.
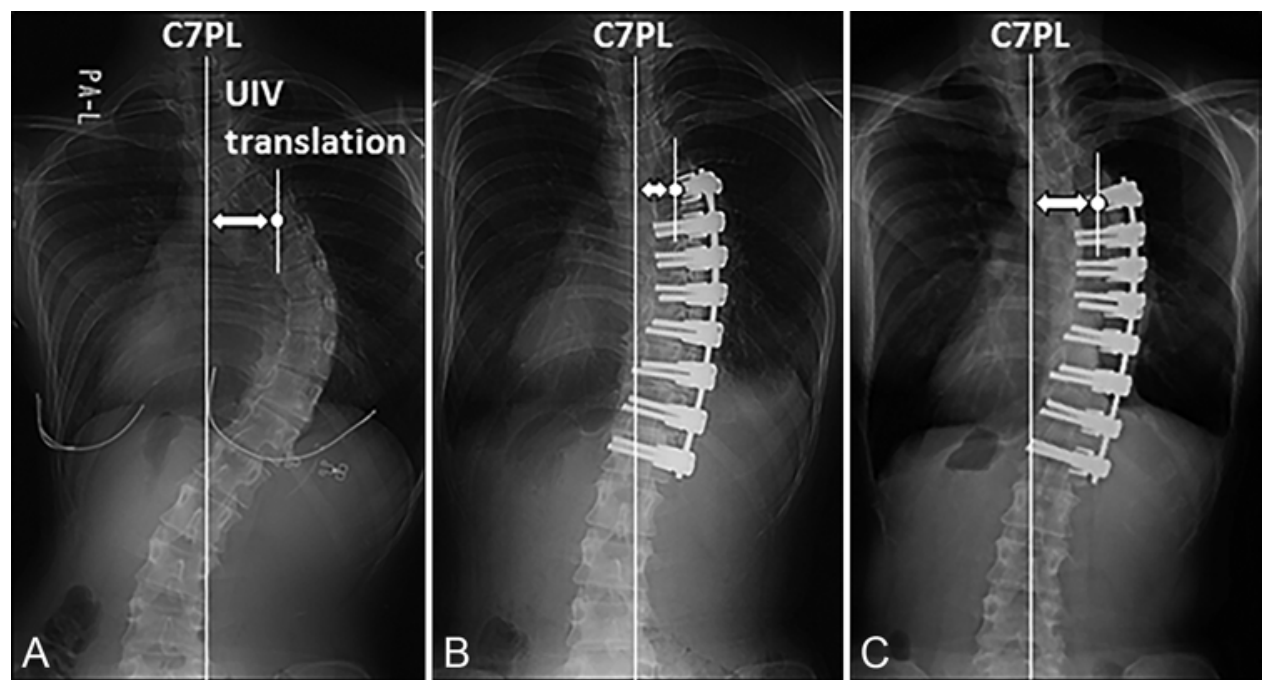

FIG. 1. Radiographs obtained in a 20 -year-old woman with UIV translation $\geq 20 \mathrm{~mm}$. The UIV translation was measured as the distance between the geometrical center of the UIV and the C7PL. The UIV translation was $51 \mathrm{~mm}$ before surgery $(\mathbf{A})$, was corrected to $27 \mathrm{~mm}$ after surgery (B), and was $45 \mathrm{~mm}$ at the final follow-up (C). 
Pulmonary function tests, both preoperatively and at the final follow-up, included the forced vital capacity $(\mathrm{FVC})$ and forced expiratory volume in 1 second $\left(\mathrm{FEV}_{1}\right)$, and these tests were conducted with the patient in a standing position. ${ }^{2}$ Each measurement was repeated three times, and the highest absolute and percent-predicted values were used for analysis.

The SRS-30 Questionnaire, which is divided into 5 domains based on the question type, was used to measure patient outcomes during the follow-up. The domains are pain, function, self-image, mental health, and satisfaction. All questions are scored from 1 to 5 (optimal); the average score for each domain was used for analysis. ${ }^{10}$

\section{Surgical Technique}

Both standing and bending films were used to select the fusion level. The instrumentation levels were determined from end-to-end vertebrae on standing films in most cases, and the vertebra without rotation on bending films was selected as the lower instrumented vertebra (LIV). ${ }^{22}$ When a neutral vertebra was located one level above the lower end vertebra (LEV), the chosen LIV was one level proximal to the LEV. Although the PT curve was corrected below $25^{\circ}$ on side bending in all patients, flexibility $<50 \%$ of the PT compensatory curve represented a rigid curve, which could cause decompensation if fused to the upper end vertebra (UEV). In such cases, one segment below the UEV was selected.

The surgical technique has been described previously. ${ }^{4,22}$ In brief, the patient was placed in the lateral decubitus position with the convex side up. After rib resection, thoracotomy was performed by incising the resected rib. After exposing the vertebral bodies, the intervertebral discs and intervening cartilage plates located within the fusion area were removed. The anterior rod was placed in the screws after securing each vertebral body in the curve with vertebral screws inserted through a vertebral staple. The rod was then rotated to decrease vertebral rotation. After resected chipped ribs were grafted into the discectomy gaps, the rod was bent to create thoracic kyphosis, and compression forces were applied between the screws to correct the scoliosis. First, compression force was applied to the anterior rod to create the kyphosis in the thoracic spine more effectively; thereafter, the posterior rod was applied in neutralization. Occasionally, it was difficult to insert 2 screws into 1 vertebral body; therefore, a 1-rod system or fixation at the proximal vertebra (9 patients), a 2-rod fixation at the distal segments (3 patients), or a combined system (13 patients) was used. Patients who were treated with a 2-rod system and were well balanced were allowed to ambulate without braces.22 For patients who showed postoperative imbalance and those treated with a 1-rod or combined system, a cervico-thoraco-lumbosacral orthosis was applied for 4-6 months after surgery. ${ }^{22}$

\section{Statistical Analysis}

All data are expressed as the mean \pm standard deviation. Independent samples t-tests (Student t-test or Welch's correction) were used to compare between-group differences in demographic data, radiographic parameters, and pulmonary function. Pearson's correlation test was used to assess the correlation of UIV translation with radiographic parameters and pulmonary function. Statistical significance was defined as $p<0.05$.

\section{Results}

The cohort was divided into two groups based on the UIV translation at the final follow-up. The group with UIV translation $<20 \mathrm{~mm}(\mathrm{n}=15)$ was defined as having UIV translation below the mean translation distance of the entire cohort $(20 \mathrm{~mm})$. The group with UIV translation $\geq 20$ $\mathrm{mm}(\mathrm{n}=10)$ was defined as having UIV translation above the mean translation distance. Among all the patients, the average age at surgery was 14.7 years (range 11-20 years), and the average Risser sign was 3.1 (range $0-5$ ). The average follow-up period was 18.2 years (range 15-21 years). The two groups were similar at baseline with respect to the following parameters: age, Risser sign, Cobb length (from UEV to LEV), instrumentation length, and followup period (Table 1).

On average, 6.5 segments were instrumented in the group with $<20 \mathrm{~mm}$ UIV translation and 6.4 segments in the group with $\geq 20 \mathrm{~mm}$ translation. The cephalad instrumented vertebra ranged from T4 to T7 (the UIV was T4 in 1 patient, T5 in 14, T6 in 8, and T7 in 2), and the caudal instrumented vertebra ranged from T10 to L2. In all patients in the $<20-\mathrm{mm}$ group, the UIV was placed at the preoperative UEV. In the $\geq 20$-mm group, 1 patient had the UIV placed below the UEV; the other 9 patients had the UIV placed at the preoperative UEV. None of the patients in either group required an extension of the fusion.

\section{Radiographic Results}

The radiographic parameters in the coronal plane are summarized in Table 2. In the preoperative and immediately postoperative periods, there was no difference in the MT curve between the groups. At the final follow-up, however, a significantly higher MT curve correction rate and lower correction loss were observed in the group with $<20$ mm UIV translation compared with those in the $\geq 20-\mathrm{mm}$ group ( $\mathrm{p}=0.010$ and 0.003 , respectively). No differences in the PT curve or global coronal balance were observed between the groups at the preoperative, immediately post-

TABLE 1. Summary of data in 25 patients surgically treated for AIS with a Lenke type 1 curve

\begin{tabular}{lccc}
\hline \multicolumn{1}{c}{ Parameter } & $\begin{array}{c}\text { UIV } \\
\text { Translation } \\
<20 \mathrm{~mm}\end{array}$ & $\begin{array}{c}\text { UIV } \\
\text { Translation } \\
\geq 20 \mathrm{~mm}\end{array}$ & $\begin{array}{c}p \\
\text { Value }\end{array}$ \\
\hline No. of patients & 15 & 10 & \\
\hline Age at surgery (yrs) & $14.4 \pm 2.5$ & $15.1 \pm 2.9$ & 0.528 \\
\hline Risser sign & $3.1 \pm 1.1$ & $3.0 \pm 1.4$ & 0.790 \\
\hline Cobb length (from UEV to LEV) & $6.6 \pm 0.7$ & $6.7 \pm 0.9$ & 0.770 \\
\hline $\begin{array}{l}\text { Instrumentation length (no. of } \\
\text { segments) }\end{array}$ & $6.5 \pm 0.6$ & $6.4 \pm 0.7$ & 0.627 \\
\hline FU period (yrs) & $18.4 \pm 1.7$ & $18.0 \pm 1.8$ & 0.579 \\
\hline FU follow-up. & & &
\end{tabular}


TABLE 2. Radiographic parameters in the coronal plane among 25 patients surgically treated for AIS with a Lenke type 1 curve

\begin{tabular}{|c|c|c|c|}
\hline Parameter & $\begin{array}{c}\text { UIV } \\
\text { Translation } \\
<20 \mathrm{~mm}\end{array}$ & $\begin{array}{c}\text { UIV } \\
\text { Translation } \\
\geq 20 \mathrm{~mm}\end{array}$ & $\begin{array}{c}p \\
\text { Value }\end{array}$ \\
\hline No. of patients & 15 & 10 & \\
\hline \multicolumn{4}{|l|}{ MT curve } \\
\hline Preoperatively $\left({ }^{\circ}\right)$ & $57.8 \pm 5.5$ & $63.8 \pm 9.3$ & 0.053 \\
\hline Flexibility (\%) & $64.9 \pm 14.6$ & $56.9 \pm 8.0$ & 0.131 \\
\hline Immediately postop $\left(^{\circ}\right)$ & $15.7 \pm 7.3$ & $19.0 \pm 7.8$ & 0.299 \\
\hline Correction rate at postop (\%) & $72.9 \pm 12.6$ & $70.4 \pm 11.6$ & 0.622 \\
\hline Final FU $\left(^{\circ}\right)$ & $22.6 \pm 8.8$ & $31.6 \pm 6.1$ & $0.010^{*}$ \\
\hline Correction rate at final FU (\%) & $61.2 \pm 14.2$ & $50.0 \pm 10.1$ & $0.010^{*}$ \\
\hline Correction loss $\left({ }^{\circ}\right)$ & $6.9 \pm 4.2$ & $12.6 \pm 4.1$ & $0.003^{*}$ \\
\hline \multicolumn{4}{|l|}{ PT curve } \\
\hline Preoperatively $\left({ }^{\circ}\right)$ & $32.6 \pm 7.2$ & $30.7 \pm 6.4$ & 0.505 \\
\hline Flexibility (\%) & $51.0 \pm 14.8$ & $55.0 \pm 20.8$ & 0.576 \\
\hline Immediately postop $\left({ }^{\circ}\right)$ & $16.5 \pm 4.6$ & $14.1 \pm 7.1$ & 0.320 \\
\hline Correction rate at postop (\%) & $48.7 \pm 13.9$ & $57.0 \pm 20.2$ & 0.234 \\
\hline Final FU $\left(^{\circ}\right)$ & $18.6 \pm 5.4$ & $17.7 \pm 6.7$ & 0.715 \\
\hline Correction rate at final FU (\%) & $41.6 \pm 17.2$ & $44.1 \pm 14.3$ & 0.712 \\
\hline \multicolumn{4}{|l|}{ Global coronal balance $(\mathrm{mm})$} \\
\hline Preoperatively & $9.4 \pm 9.4$ & $14.5 \pm 9.1$ & 0.191 \\
\hline Immediately postop & $11.5 \pm 10.9$ & $8.2 \pm 8.3$ & 0.420 \\
\hline Final FU & $9.1 \pm 5.2$ & $7.1 \pm 9.3$ & 0.505 \\
\hline \multicolumn{4}{|l|}{ AVT (mm) } \\
\hline Preoperatively & $52.7 \pm 11.8$ & $62.2 \pm 10.6$ & 0.052 \\
\hline Immediately postop & $11.8 \pm 11.7$ & $18.9 \pm 10.9$ & 0.140 \\
\hline Final FU & $20.5 \pm 5.9$ & $35.2 \pm 7.8$ & $<0.001^{*}$ \\
\hline \multicolumn{4}{|l|}{ Chest cage ratio } \\
\hline Preoperatively & $1.8 \pm 0.2$ & $1.9 \pm 0.3$ & 0.248 \\
\hline Immediately postop & $1.3 \pm 0.2$ & $1.4 \pm 0.1$ & 0.462 \\
\hline Final FU & $1.3 \pm 0.1$ & $1.5 \pm 0.1$ & $0.005^{*}$ \\
\hline \multicolumn{4}{|l|}{$\mathrm{RSH}(\mathrm{mm})$} \\
\hline Preoperatively & $-5.1 \pm 8.7$ & $-7.8 \pm 7.0$ & 0.436 \\
\hline Immediately postop & $3.3 \pm 8.1$ & $-1.6 \pm 12.2$ & 0.256 \\
\hline Final FU & $2.0 \pm 8.9$ & $-8.0 \pm 11.5$ & $0.025^{*}$ \\
\hline
\end{tabular}

* Statistically significant.

operative, and final follow-up time points. With regard to AVT, chest cage ratio, and RSH, there were no differences between the groups in either the preoperative or immediately postoperative period. At the final follow-up, however, the $\geq 20$-mm group had a significantly larger AVT, higher chest cage ratio, and lower RSH than the $<20$-mm group $(\mathrm{p}<0.001, \mathrm{p}=0.005$, and $\mathrm{p}=0.025$, respectively).

Radiographic parameters in the upper adjacent segments are summarized in Table 3 . The $\geq 20-\mathrm{mm}$ group showed significantly higher UIV translation than the < 20 -mm group at the preoperative, immediately postoperative, and final follow-up time points $(\mathrm{p}<0.001, \mathrm{p}=0.002$, and $p<0.001$, respectively). No significant differences in the preoperative UIV tilt or UIV disc angle were found
TABLE 3. Radiographic parameters in the upper adjacent segments in 25 patients surgically treated for AIS with a Lenke type 1 curve

\begin{tabular}{lccc}
\hline \multicolumn{1}{c}{ Parameter } & $\begin{array}{c}\text { UIV Translation } \\
<20 \mathrm{~mm}\end{array}$ & $\begin{array}{c}\text { UIV Translation } \\
\geq 20 \mathrm{~mm}\end{array}$ & $\begin{array}{c}\mathrm{p} \\
\text { Value }\end{array}$ \\
\hline No. of patients & 15 & 10 & \\
\hline UIV translation $(\mathrm{mm})$ & & & \\
\hline Preoperatively & $21.9 \pm 7.7$ & $36.8 \pm 9.2$ & $<0.001^{*}$ \\
\hline Immediately postop & $6.0 \pm 6.4$ & $16.3 \pm 8.3$ & $0.002^{*}$ \\
\hline Final FU & $12.6 \pm 4.9$ & $30.0 \pm 7.5$ & $<0.001^{*}$ \\
\hline UIV tilt $\left(^{\circ}\right)$ & & & \\
\hline Preoperatively & $29.9 \pm 4.9$ & $32.7 \pm 4.9$ & 0.200 \\
\hline Immediately postop & $8.3 \pm 3.9$ & $10.4 \pm 4.5$ & 0.238 \\
\hline Final FU & $14.6 \pm 4.1$ & $20.8 \pm 4.2$ & $0.001^{*}$ \\
\hline UIV disc angle $\left(^{\circ}\right)$ & & & \\
\hline Preoperatively & $2.2 \pm 1.7$ & $1.7 \pm 2.5$ & 0.560 \\
\hline Immediately postop & $-2.2 \pm 1.1$ & $-3.2 \pm 1.8$ & 0.104 \\
\hline Final FU & $-1.9 \pm 0.9$ & $-3.5 \pm 2.7$ & $0.047^{*}$ \\
\hline
\end{tabular}

* Statistically significant.

between the two groups. At the final follow-up, however, the group with $\geq 20 \mathrm{~mm}$ UIV translation showed a significantly larger UIV tilt and lower UIV disc angle than the < 20 -mm group ( $\mathrm{p}=0.001$ and 0.047 , respectively).

Radiographic parameters in the sagittal plane are summarized in Table 4. Preoperative instrumented level kyphosis was significantly higher in the $<20$-mm group than in the $\geq 20$-mm group ( $p=0.016$ ). At the final follow-up, however, no significant difference regarding instrumented level kyphosis was found between the two groups. There was no significant difference in the proximal junctional kyphosis and global sagittal balance between the groups at the preoperative, immediately postoperative, and final follow-up time points.

\section{Pulmonary Function Results}

Pulmonary function data are presented in Table 5. Clinically, no patients had complaints related to pulmonary function. At the preoperative or final follow-up assessments, no difference in the FVC, percent-predicted $\mathrm{FVC}$, or $\mathrm{FEV}_{1}$ was found between the groups. The percentpredicted $\mathrm{FEV}_{1}$ decreased at the final follow-up in each group. Preoperatively, the group with UIV translation $\geq 20$ $\mathrm{mm}$ had a significantly lower percent-predicted $\mathrm{FEV}_{1}$ than the group with translation $<20 \mathrm{~mm}(\mathrm{p}=0.046)$. At the final follow-up, however, no significant difference in the percent-predicted $\mathrm{FEV}_{1}$ was observed between the groups.

\section{Correlation Analysis}

Correlation analysis was conducted between the UIV translation values and radiographic parameters at the final follow-up (Table 6). The UIV translation was significantly correlated with the MT curve correction rate and correction loss $(\mathrm{r}=-0.481, \mathrm{p}=0.015 ; \mathrm{r}=0.463, \mathrm{p}=0.020$, respectively). Similarly, UIV translation was significantly correlated with the AVT and chest cage ratio $(r=0.844, p$ 
TABLE 4. Radiographic parameters in the sagittal plane in 25 patients surgically treated for AIS with a Lenke type 1 curve

\begin{tabular}{lccc}
\hline \multicolumn{1}{c}{ Parameter } & $\begin{array}{c}\text { UIV } \\
\text { Translation } \\
<20 \mathrm{~mm}\end{array}$ & $\begin{array}{c}\text { UIV } \\
\text { Translation } \\
\geq 20 \mathrm{~mm}\end{array}$ & $\begin{array}{c}\mathrm{p} \\
\text { Value }\end{array}$ \\
\hline No. of patients & 15 & 10 & \\
\hline Instrumented level kyphosis $\left(^{\circ}\right)$ & & & \\
\hline Preoperatively & $12.8 \pm 10.8$ & $1.5 \pm 10.5$ & $0.016^{*}$ \\
\hline Immediately postop & $17.5 \pm 5.6$ & $13.2 \pm 6.2$ & 0.083 \\
\hline Final FU & $20.3 \pm 6.0$ & $16.1 \pm 5.6$ & 0.091 \\
\hline Proximal junctional kyphosis $\left(^{\circ}\right)$ & & & \\
\hline Preoperatively & $1.2 \pm 0.4$ & $1.2 \pm 0.4$ & 1.000 \\
\hline Immediately postop & $1.9 \pm 1.0$ & $1.6 \pm 1.1$ & 0.426 \\
\hline Final FU & $2.1 \pm 1.0$ & $2.8 \pm 1.0$ & 0.137 \\
\hline Sagittal vertical axis $(\mathrm{mm})$ & & & \\
\hline Preoperatively & $6.3 \pm 17.6$ & $8.9 \pm 15.1$ & 0.710 \\
\hline Immediately postop & $10.8 \pm 17.1$ & $20.2 \pm 20.8$ & 0.229 \\
\hline Final FU & $4.5 \pm 23.0$ & $16.1 \pm 20.6$ & 0.213 \\
\hline
\end{tabular}

* Statistically significant.

$<0.001 ; \mathrm{r}=0.673, \mathrm{p}<0.001$, respectively). Conversely, no correlation was found between UIV translation and RSH. In addition, correlation analysis was conducted between UIV translation and pulmonary function at the final follow-up. A significant correlation was found between UIV translation and the percent-predicted $\mathrm{FEV}_{1}$ at the final follow-up $(\mathrm{r}=-0.455, \mathrm{p}=0.033)$.

\section{SRS-30 Questionnaire Data}

The SRS-30 Questionnaire total and individual domain scores at the final follow-up are shown in Table 7. The mean total score was 4.1 in the group with UIV translation $<20 \mathrm{~mm}$ and 3.9 in the group with $\geq 20 \mathrm{~mm}$ UIV

TABLE 5. Pulmonary function in 25 patients surgically treated for AIS with a Lenke type 1 curve

\begin{tabular}{cccc}
\hline Parameter & $\begin{array}{c}\text { UIV Translation } \\
<20 \mathrm{~mm}\end{array}$ & $\begin{array}{c}\text { UIV Translation } \\
\geq 20 \mathrm{~mm}\end{array}$ & $\begin{array}{c}\mathrm{p} \\
\text { Value }\end{array}$ \\
\hline No. of patients & 15 & 10 & \\
\hline FVC $(\mathrm{L})$ & & & \\
\hline Preoperatively & $2.4 \pm 0.3$ & $2.5 \pm 0.5$ & 0.299 \\
\hline Final FU & $2.4 \pm 0.4$ & $2.5 \pm 0.7$ & 0.539 \\
\hline$\%$ predicted FVC & & & \\
\hline Preoperatively & $82.6 \pm 13.1$ & $82.3 \pm 14.2$ & 0.956 \\
\hline Final FU & $74.6 \pm 11.4$ & $72.4 \pm 10.9$ & 0.658 \\
\hline FEV ${ }_{1}(\mathrm{~L})$ & & & \\
\hline Preoperatively & $2.1 \pm 0.3$ & $2.0 \pm 0.4$ & 0.555 \\
\hline Final FU & $2.1 \pm 0.3$ & $2.1 \pm 0.7$ & 0.862 \\
\hline$\%$ predicted FEV ${ }_{1}$ & & & \\
\hline Preoperatively & $83.6 \pm 12.2$ & $73.6 \pm 8.9$ & $0.046^{*}$ \\
\hline Final FU & $73.2 \pm 9.4$ & $65.4 \pm 12.0$ & 0.102 \\
\hline
\end{tabular}

* Statistically significant.
TABLE 6. Correlation analysis between UIV translation and other variables at the final follow-up

\begin{tabular}{lcc}
\hline \multicolumn{1}{c}{ Variable } & Correlation Coefficient $(r)$ & $p$ Value \\
\hline Radiographic parameters & & \\
\hline Correction rate & -0.481 & $0.015^{*}$ \\
\hline Correction loss & 0.463 & $0.020^{*}$ \\
\hline AVT & 0.844 & $<0.001^{*}$ \\
\hline Chest cage ratio & 0.673 & $<0.001^{*}$ \\
\hline RSH & -0.274 & 0.195 \\
\hline Pulmonary function & & \\
\hline FVC & -0.024 & 0.916 \\
\hline$\%$ predicted FVC & -0.267 & 0.230 \\
\hline FEV & -0.172 & 0.444 \\
\hline$\%$ predicted FEV & -0.455 & $0.033^{*}$ \\
\hline
\end{tabular}

* Statistically significant.

translation. There was no significant difference between the groups in the total or individual domain scores.

\section{Discussion}

Although selection of the LIV is thought to be an important factor affecting distal junctional problems or postoperative global imbalance,,$^{12}$ and even though radiographic parameters such as RSH, T1 tilt, and UIV tilt are established parameters indicating shoulder imbalance and a proximal adding-on effect, UIV translation has not been fully discussed because few studies have focused on the selection of the UIV. In the current study, we demonstrated that a large UIV translation from the C7PL is associated with a lower correction rate of the MT curve, lower $\mathrm{RSH}$, and diminished pulmonary function. These results suggest that selection of the UIV is also an important factor influencing postoperative results.

In PSF for thoracic AIS, it is recommended that the UIV be fused to T2, T3, T4, or T5, depending on the shoulder level and flexibility of the PT curve. ${ }^{25}$ Conversely, selection of the UIV is controversial in ASF for Lenke type 1 AIS, although the procedure can provide short fusion within end-to-end vertebrae. Anterior spinal fusion for thoracic scoliosis limits the selection of the PT vertebrae,

TABLE 7. SRS-30 clinical scores at the final follow-up

\begin{tabular}{lccc}
\hline \multicolumn{1}{c}{ Parameter } & $\begin{array}{c}\text { UIV } \\
\text { Translation } \\
<20 \mathrm{~mm}\end{array}$ & $\begin{array}{c}\text { UIV } \\
\text { Translation } \\
\geq 20 \mathrm{~mm}\end{array}$ & $\begin{array}{c}\mathrm{p} \\
\text { Value }\end{array}$ \\
\hline No. of patients & 15 & 10 & \\
\hline Score & & & \\
\hline Function/activity & $4.4 \pm 0.3$ & $4.2 \pm 0.3$ & 0.124 \\
\hline Pain & $4.3 \pm 0.4$ & $4.3 \pm 0.5$ & 0.841 \\
\hline Self-image/appearance & $3.6 \pm 0.5$ & $3.5 \pm 0.6$ & 0.469 \\
\hline Mental health & $4.2 \pm 0.6$ & $3.8 \pm 0.6$ & 0.210 \\
\hline Satisfaction w/ management & $3.9 \pm 0.7$ & $3.6 \pm 0.5$ & 0.332 \\
\hline Total & $4.1 \pm 0.3$ & $3.9 \pm 0.4$ & 0.200 \\
\hline
\end{tabular}


for example, using T1 to T3 for the UIV, because instrumentation to the upper thoracic vertebrae is very difficult given the presence of the scapular arch. Consequently, the UIV is usually selected at or below the preoperative UEV. In this study, the UIV was placed at the preoperative UEV in all patients except for one whose UIV was placed below the UEV. Although the level of the UIV was identical to that of the UEV in most cases, UIV translation became larger over the long follow-up period in some cases.

Upper instrumented vertebra translation may be a factor influencing postoperative results. Liu et al. reported that Lenke type 5C thoracolumbar/lumbar AIS patients who had greater UIV translation before ASF surgery might have a higher risk of developing immediate postoperative coronal imbalance. ${ }^{9}$ Furthermore, these authors found that postoperative UIV translation was significantly correlated with immediate postoperative coronal balance. In the present study, at the final follow-up, patients with a UIV translation $\geq 20 \mathrm{~mm}$ showed a lower MT curve correction rate and lower $\mathrm{RSH}$ than those with a UIV translation $<20 \mathrm{~mm}$. Furthermore, UIV translation had a significant negative correlation with pulmonary function at the final follow-up. However, no differences in global balance were observed between the groups, and patients with UIV translation $\geq 20 \mathrm{~mm}$ had a mean C7PL-CSVL distance within $10 \mathrm{~mm}$. This is because the UIV tilt was increased by approximately $10^{\circ}$ in the group with UIV translation $\geq$ $20 \mathrm{~mm}$, compensating for coronal imbalance. ${ }^{9}$

Because the patients with a UIV translation $\geq 20 \mathrm{~mm}$ at the final follow-up had had a significantly larger preoperative UIV translation than the patients whose final follow-up UIV translation was $<20 \mathrm{~mm}$, preoperative UIV translation may be a predictive factor for a large UIV translation at the postoperative follow-up. In addition, a significant correlation was found between preoperative UIV translation and the MT curve at the final follow-up ( $\mathrm{r}=0.482, \mathrm{p}=0.017)$, indicating that preoperative UIV translation should be considered an important factor influencing the postoperative MT curve. We cannot suggest a strict baseline for acceptable UIV translation because this study was an intergroup trial. However, in MT AIS patients whose preoperative UEV is distant from the C7PL, the UIV may need to be placed above the UEV to decrease UIV translation because the preoperative UIV translation can be smaller when the UIV is selected above the UEV than when the UIV is selected at the UEV.

Selection of the UIV may also be important for preventing the proximal adding-on effect. Nohara et al. reported that 10 years after ASF surgery, disc wedging $>5^{\circ}$ at segments adjacent to the UIV was found in $27 \%$ of thoracic AIS patients whose UIV had been placed at or below the preoperative UEV. ${ }^{15}$ However, there was no proximal adding-on effect in the PSF patients, all of whom had had the UIV placed above the preoperative UEV except for one patient whose UIV had been placed at the preoperative UEV. ${ }^{15}$ In the current study, the UIV disc angle in both groups was $<5^{\circ}$ at the final follow-up, and this was considered as not clinically significant. However, the group with UIV translation $\geq 20 \mathrm{~mm}$ showed a significantly lower UIV disc angle than that in the group with $<20 \mathrm{~mm}$ UIV translation at the final follow-up, suggesting that UIV translation may be a factor influencing UIV disc wedging and the proximal adding-on effect.

An open anterior approach is associated with a decline in pulmonary function..$^{14,21,22}$ In the current study, a significant negative correlation was found between UIV translation and the percent-predicted $\mathrm{FEV}_{1}$. In addition, the group with $\geq 20 \mathrm{~mm}$ UIV translation had a significantly higher chest cage imbalance, indicating that a large UIV translation was associated with chest cage imbalance, which may be associated with a decrease in pulmonary function.

When examining patient-based outcomes, we found no significant difference in SRS-30 scores between the groups at the final follow-up perhaps because most patients achieved good correction of the MT curve and global coronal balance, and the shoulder imbalance was not severe enough to impact patient satisfaction with the results of surgery. ${ }^{13}$

\section{Conclusions}

More selective ASF has been touted as offering similarto-superior outcomes in terms of scoliosis correction, as compared with less selective PSF. However, an increase in the UIV translation after ASF for Lenke type 1 AIS was significantly correlated with MT curve correction loss and decreased pulmonary function, indicating that UIV translation should be considered an important factor influencing postoperative results. Selection of the UIV is critical as it can lead to decompensation later in life. In Lenke type 1 MT AIS patients, whose preoperative UEV is distant from the C7PL, surgeons would likely consider extending the UIV above the UEV to prevent a large UIV translation at the postoperative follow-up. In other words, when the UEV for a relatively rigid and moderate-to-severe type 1 AIS is at or above T4, correction involving ASF will be difficult. Thus, the UIV above the UEV level may be chosen (i.e., T3, T2, or T1); however, fusion will be very challenging owing to the scapular arch. In such cases, PSF will most likely be considered.

\section{References}

1. Brodner W, Mun Yue W, Möller HB, Hendricks KJ, Burd TA, Gaines RW: Short segment bone-on-bone instrumentation for single curve idiopathic scoliosis. Spine (Phila Pa 1976) 28:S224-S233, 2003

2. Gitelman Y, Lenke LG, Bridwell KH, Auerbach JD, Sides BA: Pulmonary function in adolescent idiopathic scoliosis relative to the surgical procedure: a 10-year follow-up analysis. Spine (Phila Pa 1976) 36:1665-1672, 2011

3. Jackson RP, McManus AC: Radiographic analysis of sagittal plane alignment and balance in standing volunteers and patients with low back pain matched for age, sex, and size. A prospective controlled clinical study. Spine (Phila Pa 1976) 19:1611-1618, 1994

4. Kaneda K, Shono Y, Satoh S, Abumi K: Anterior correction of thoracic scoliosis with Kaneda anterior spinal system. A preliminary report. Spine (Phila Pa 1976) 22:1358-1368, 1997

5. Kelly DM, McCarthy RE, McCullough FL, Kelly HR: Longterm outcomes of anterior spinal fusion with instrumentation for thoracolumbar and lumbar curves in adolescent idiopathic scoliosis. Spine (Phila Pa 1976) 35:194-198, 2010

6. Kim YJ, Lenke LG, Cho SK, Bridwell KH, Sides B, Blanke 
$\mathrm{K}$ : Comparative analysis of pedicle screw versus hook instrumentation in posterior spinal fusion of adolescent idiopathic scoliosis. Spine (Phila Pa 1976) 29:2040-2048, 2004

7. Lenke LG, Betz RR, Harms J, Bridwell KH, Clements DH, Lowe TG, et al: Adolescent idiopathic scoliosis: a new classification to determine extent of spinal arthrodesis. J Bone Joint Surg Am 83-A:1169-1181, 2001

8. Liljenqvist UR, Bullmann V, Schulte TL, Hackenberg L, Halm HF: Anterior dual rod instrumentation in idiopathic thoracic scoliosis. Eur Spine J 15:1118-1127, 2006

9. Liu Z, Guo J, Zhu Z, Qian B, Sun X, Xu L, et al: Role of the upper and lowest instrumented vertebrae in predicting the postoperative coronal balance in Lenke $5 \mathrm{C}$ patients after selective posterior fusion. Eur Spine J 22:2392-2398, 2013

10. Lubicky JP, Hanson JE, Riley EH: Instrumentation constructs in pediatric patients undergoing deformity correction correlated with Scoliosis Research Society scores. Spine (Phila Pa 1976) 36:1692-1700, 2011

11. Luhmann SJ, Sucato DJ, Johnston CE, Richards BS, Karol LA: Radiographic assessment of shoulder position in 619 idiopathic scoliosis patients: can T1 tilt be used as an intraoperative proxy to determine postoperative shoulder balance? J Pediatr Orthop 36:691-694, 2016

12. Matsumoto M, Watanabe K, Hosogane N, Kawakami N, Tsuji T, Uno K, et al: Postoperative distal adding-on and related factors in Lenke type 1A curve. Spine (Phila Pa 1976) 38:737-744, 2013

13. Matsumoto M, Watanabe K, Kawakami N, Tsuji T, Uno K, Suzuki T, et al: Postoperative shoulder imbalance in Lenke Type 1A adolescent idiopathic scoliosis and related factors. BMC Musculoskelet Disord 15:366, 2014

14. Newton PO, Marks MC, Bastrom TP, Betz R, Clements D, Lonner B, et al: Surgical treatment of Lenke 1 main thoracic idiopathic scoliosis: results of a prospective, multicenter study. Spine (Phila Pa 1976) 38:328-338, 2013

15. Nohara A, Kawakami N, Saito T, Tsuji T, Ohara T, Suzuki Y, et al: Comparison of surgical outcomes between anterior fusion and posterior fusion in patients with AIS Lenke type 1 or 2 that underwent selective thoracic fusion-long-term follow-up study longer than 10 postoperative years. Spine (Phila Pa 1976) 40:1681-1689, 2015

16. Potter BK, Kuklo TR, Lenke LG: Radiographic outcomes of anterior spinal fusion versus posterior spinal fusion with thoracic pedicle screws for treatment of Lenke Type I adolescent idiopathic scoliosis curves. Spine (Phila Pa 1976) 30:1859-1866, 2005

17. Saraph VJ, Krismer M, Wimmer C: Operative treatment of scoliosis with the Kaneda anterior spine system. Spine (Phila Pa 1976) 30:1616-1620, 2005

18. Satake K, Lenke LG, Kim YJ, Bridwell KH, Blanke KM, Sides B, et al: Analysis of the lowest instrumented vertebra following anterior spinal fusion of thoracolumbar/lumbar adolescent idiopathic scoliosis: can we predict postoperative disc wedging? Spine (Phila Pa 1976) 30:418-426, 2005

19. Schwab F, Patel A, Ungar B, Farcy JP, Lafage V: Adult spinal deformity-postoperative standing imbalance: how much can you tolerate? An overview of key parameters in assessing alignment and planning corrective surgery. Spine (Phila Pa 1976) 35:2224-2231, 2010

20. Sudo H, Abe Y, Kokabu T, Ito M, Abumi K, Ito YM, et al: Correlation analysis between change in thoracic kyphosis and multilevel facetectomy and screw density in main thoracic adolescent idiopathic scoliosis surgery. Spine J 16:10491054,2016

21. Sudo H, Ito M, Kaneda K, Shono Y, Abumi K: Long-term outcomes of anterior dual-rod instrumentation for thoracolumbar and lumbar curves in adolescent idiopathic scoliosis: a twelve to twenty-three-year follow-up study. J Bone Joint Surg Am 95:e49, 2013

22. Sudo H, Ito M, Kaneda K, Shono Y, Takahata M, Abumi K: Long-term outcomes of anterior spinal fusion for treating thoracic adolescent idiopathic scoliosis curves: average 15year follow-up analysis. Spine (Phila Pa 1976) 38:819-826, 2013

23. Sudo H, Kaneda K, Shono Y, Iwasaki N: Selection of the upper vertebra to be instrumented in the treatment of thoracolumbar and lumbar adolescent idiopathic scoliosis by anterior correction and fusion surgery using dual-rod instrumentation: a minimum 12-year follow-up study. Spine J 16:281-287, 2016

24. Tis JE, O'Brien MF, Newton PO, Lenke LG, Clements DH, Harms J, et al: Adolescent idiopathic scoliosis treated with open instrumented anterior spinal fusion: five-year follow-up. Spine (Phila Pa 1976) 35:64-70, 2010

25. Trobisch PD, Ducoffe AR, Lonner BS, Errico TJ: Choosing fusion levels in adolescent idiopathic scoliosis. J Am Acad Orthop Surg 21:519-528, 2013

\section{Disclosures}

The authors report no conflict of interest concerning the materials or methods used in this study or the findings specified in this paper.

\section{Author Contributions}

Conception and design: Sudo. Acquisition of data: Sudo, Shono. Analysis and interpretation of data: Sudo, Yamada, Abe. Drafting the article: Sudo, Yamada. Critically revising the article: Sudo. Reviewed submitted version of manuscript: Sudo, Kaneda, Shono, Abe, Iwasaki. Approved the final version of the manuscript on behalf of all authors: Sudo. Statistical analysis: Sudo. Administrative/technical/material support: Kaneda. Study supervision: Sudo, Iwasaki.

\section{Correspondence}

Hideki Sudo: Hokkaido University Graduate School of Medicine, Sapporo, Hokkaido, Japan. hidekisudo@yahoo.co.jp. 